\title{
Two-photon instant structured illumination microscopy improves the depth penetration of super-resolution imaging in thick, scattering samples: supplementary
}

\section{material}

PETER W. WINTER ${ }^{1 *}$, ANDREW G. YORK ${ }^{1}$, DAMIAN DALLE NOGARE ${ }^{2}$, MARIA INGARAMO ${ }^{3}$, RYAN CHRISTENSEN ${ }^{1}$, AJAY CHITNIS ${ }^{2}$, GEORGE H. PATTERSON ${ }^{3}$, HARI SHROFF ${ }^{1}$

${ }^{1}$ Section on High Resolution Optical Imaging, National Institute of Biomedical Imaging and Bioengineering, National Institutes of Health, Bethesda, MD, USA

${ }^{2}$ Section on Neural Developmental Dynamics, Eunice Kennedy Shriver National Institute of Child Health and Human Development, National Institutes of Health, Bethesda, MD, USA

${ }^{3}$ Section on Biophotonics, National Institute of Biomedical Imaging and Bioengineering, National Institutes of Health, Bethesda, MD, USA

*Correspondence to peter.winter@nih.gov

Published 12 September 2014

This document provides supplementary information to "Two-photon instant structured illumination microscopy improves the depth penetration of super-resolution imaging in thick, scattering samples," http://dx.doi.org/10.1364/OPTICA.1.000181. This includes additional data, imaging parameters, media captions and notes.

http://dx.doi.org/10.1364/0PTICA.1.000181.soo1

Media 1: 2P ISIM imaging of live transgenic C. elegans embryo expressing GFP-H2B. The stack was acquired with 250 nm z-steps spanning a thickness of $\sim 30 \mu \mathrm{m}$. Data are deconvolved. See also Fig. 4.

Media 2: 1P ISIM imaging of live transgenic C. elegans embryo expressing GFP-H2B. The stack was acquired with 250 $\mathrm{nm}$ z-steps spanning a thickness of $\sim 30 \mu \mathrm{m}$. Data are deconvolved. See also Fig. S11.

Media 3: 3D rendering of 2P ISIM imaging of live transgenic C. elegans $\mathrm{L} 2$ larva expressing transcriptional reporter psax-3::GFP. The stack was acquired with $500 \mathrm{~nm}$ z-steps spanning a thickness of $\sim 25 \mu \mathrm{m}$. Note that only the GFP channel is shown. Due to space limitations data were down-sampled to $75 \mathrm{~nm}$ per pixel. Data are deconvolved. See also Fig. 5.

Media 4: 2P ISIM imaging of eyeball of live transgenic zebrafish embryo expressing GFP-tagged microtubules. The stack was acquired with $250 \mathrm{~nm}$ z-steps spanning a thickness of $\sim 110 \mu \mathrm{m}$. The first $\sim 8 \mu \mathrm{m}$ of the image stack is agarose between the coverslip and the fish embryo. Due to space limitations data were down-sampled to $75 \mathrm{~nm}$ per pixel. Data are deconvolved. See also Fig. 6. 
Media 5: 3D rendering of 2P ISIM imaging of eyeball of live transgenic zebrafish embryo expressing GFP-tagged

microtubules. The stack was acquired with $250 \mathrm{~nm}$ z-steps spanning a thickness of $\sim 110 \mu \mathrm{m}$. Data are deconvolved. See also Fig. 6 and Fig. S13.

Media 6: 2P ISIM imaging of midbrain of live transgenic zebrafish embryo expressing GFP-tagged microtubules. The stack was acquired with $250 \mathrm{~nm}$ z-steps spanning a $\sim 70 \mu \mathrm{m}$ thickness. Due to space limitations data were down-sampled to 75 nm per pixel. See also Fig. 7.

Media 7: Conventional 2P imaging of midbrain of live transgenic zebrafish embryo expressing GFP-tagged

microtubules. The stack was acquired with $250 \mathrm{~nm}$ z-steps spanning a thickness of $\sim 70 \mu \mathrm{m}$. Due to space limitations data were down-sampled to $75 \mathrm{~nm}$ per pixel. See also Fig. 7.

Fig. S1, Equivalent schemes for enhancing resolution in point-based SIM.

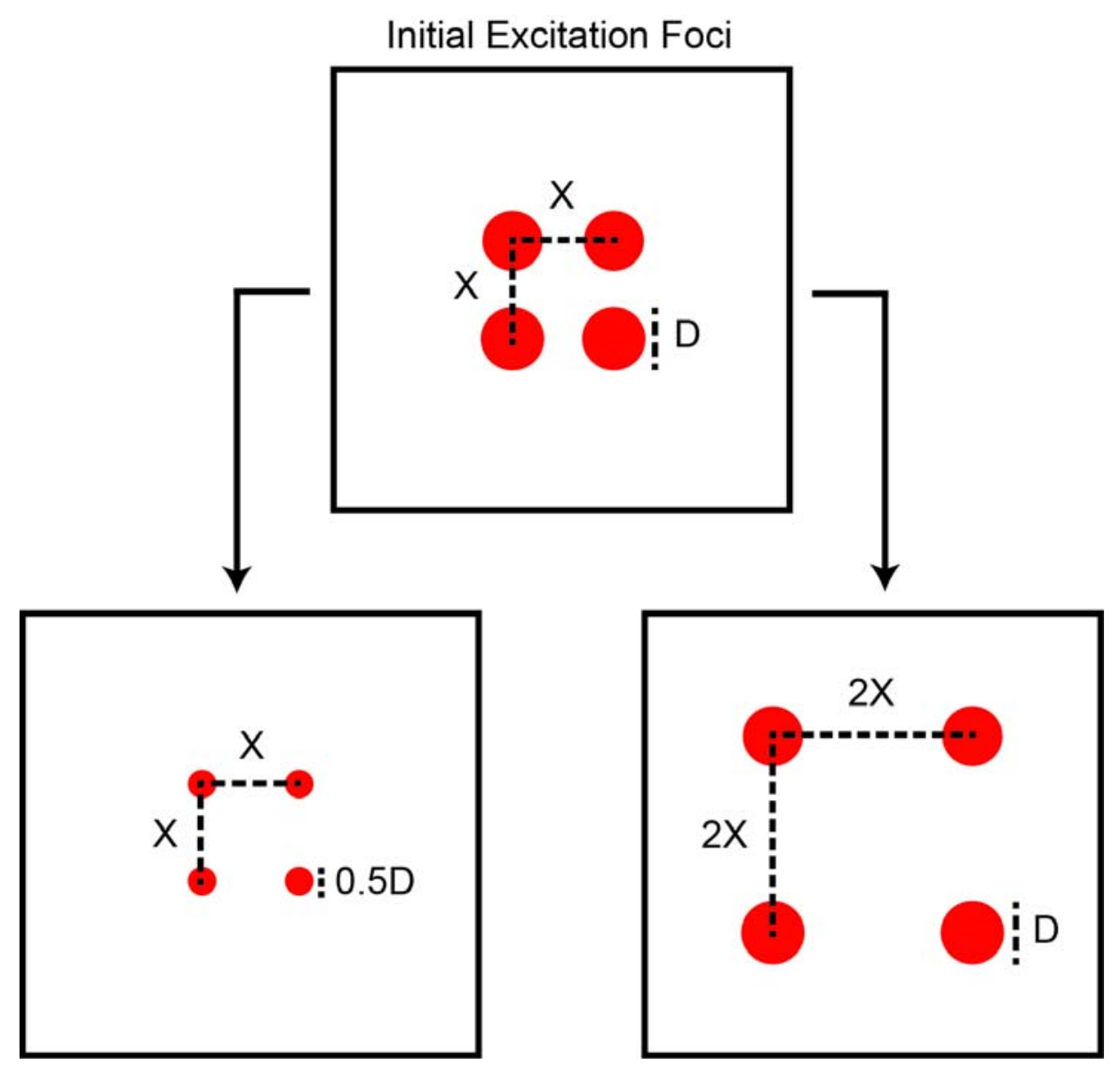

Top: Excitation foci (red circles) are created by rastering an excitation focus across the sample. Here, 4 excitation foci are shown with inter-focus distance X and diameter D. Bottom: Two methods for increasing the resolution of the resulting image. In previous the instant SIM, foci are shrunk without altering the distance between them (left). In this work, we use a second, emission side galvanometric mirror to increase the inter-foci distance to $2 \mathrm{X}$, while leaving the size of the foci unchanged (right). Either method produces an equivalent result, as the only difference between the output images is a global scaling factor. 
Fig. S2, Example galvo control waveforms.

(a)

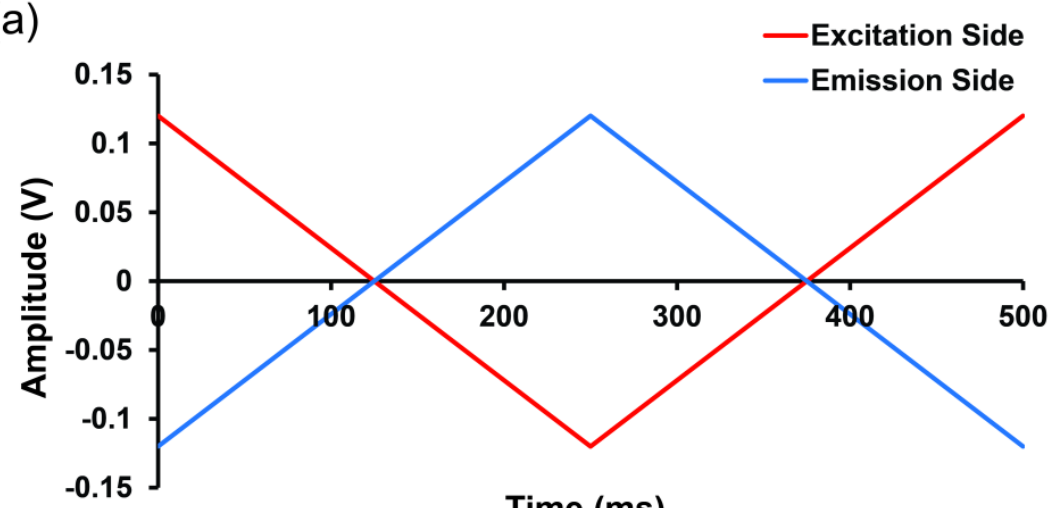

(b)

Time (ms)

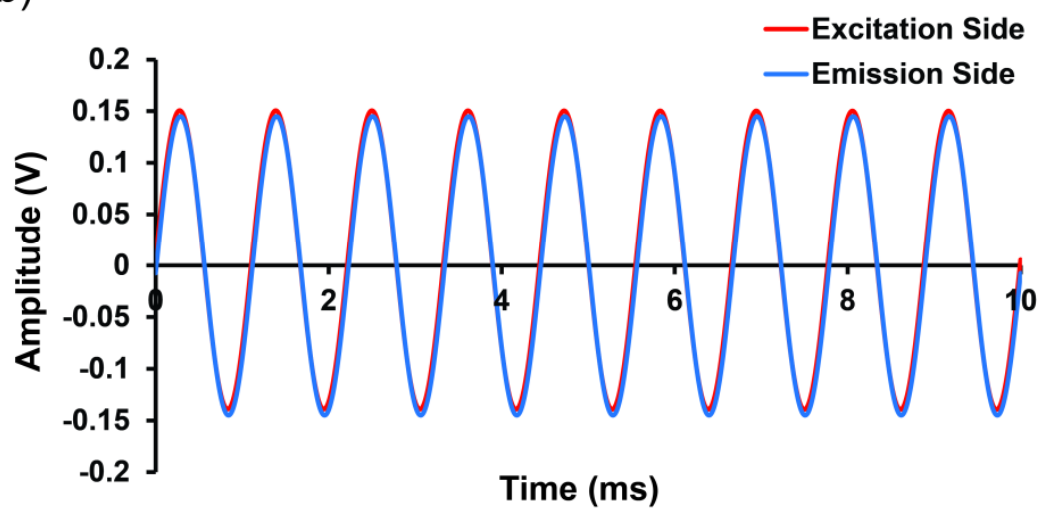

(c)

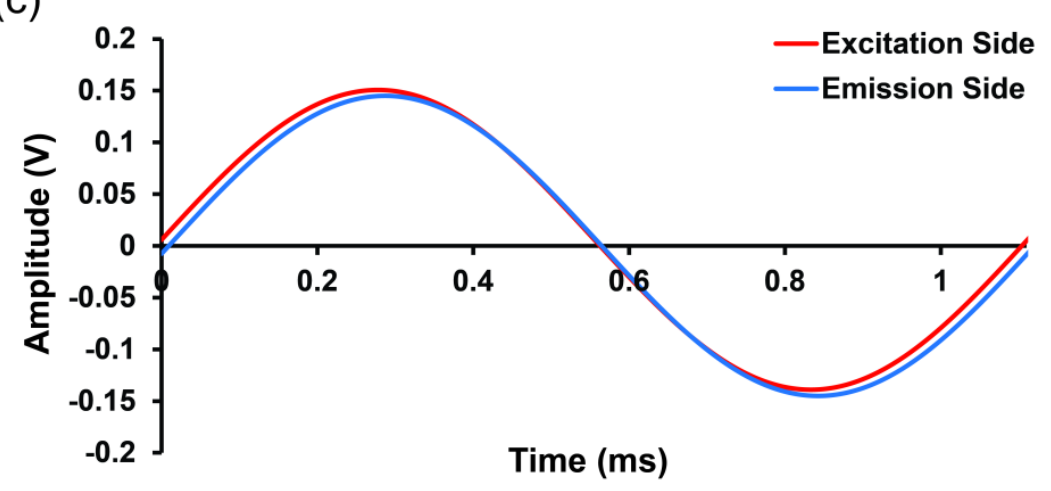

Example analog waveforms produced by the DAQ card to control movement of the 2D galvos. In (a) both excitation- and emission-side 'slow' axis galvos are scanned using a triangle wave at $2 \mathrm{~Hz}$. In (b) both excitation- and emission-side 'fast' axis galvos are scanned at $900 \mathrm{~Hz}$. In (c) we show a higher magnification view of a single cycle in (b) emphasizing the slight phase offset needed to achieve optimal imaging. See also Fig. S7. 
Fig. S3, Example hardware control waveforms.
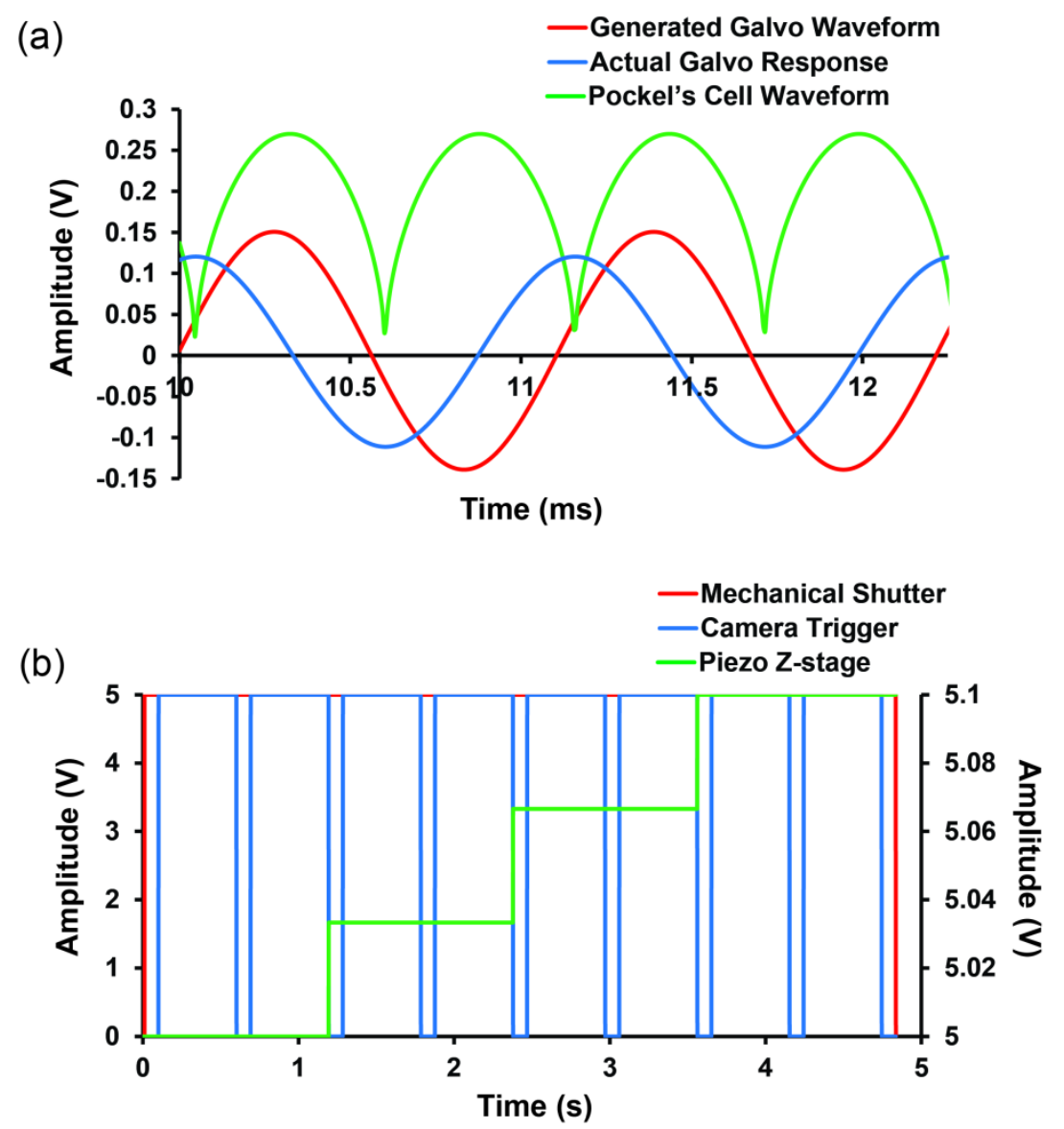

(a) Example of the synchronization of the Pockels cell correction waveform to the actual position of the fast galvo position (for clarity the position of only the emission side galvo is shown). The actual response of the galvo significantly lags behind the control waveform produced by the DAQ, and thus the actual range of travel of the fast galvo is slightly less than would be expected given the delivered waveform. (b) Example of the control waveforms required to control the mechanical shutter, camera and piezo stage for a 3D dataset with 4 z-steps separated by $1 \mu \mathrm{m}$ each with 2 frame averaging per plane. 
Fig. S4, Correcting intensity variation across the field of view.

(a)

(b)
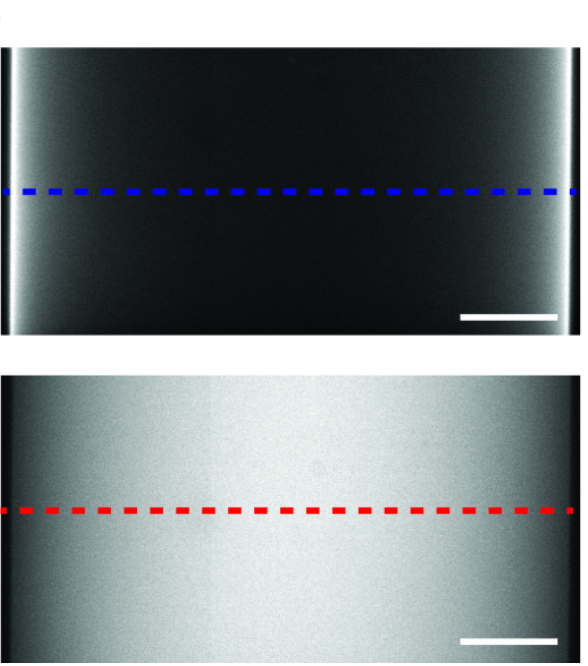

(c)

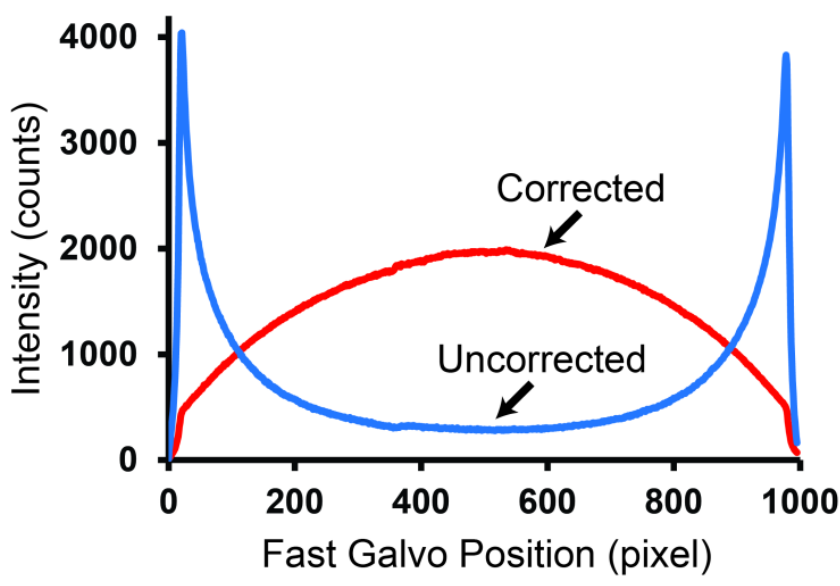

(a) Fluorescence signal recorded across the field of view when scanning the fast galvo in a sinusoidal pattern, while holding the illumination intensity constant (shown on a thick fluorescent lake). As the fast galvo changes direction at the end of each scan it must slow down resulting in a significant increase in the total 2P illumination dose delivered to the sample. (b) Fluorescence signal recorded after compensating for the changes in fast galvo speed across the field of view. Correction is achieved by applying a square-root cosine function to the Pockels cell to modulate illumination intensity during the scan. (c) Line profiles through dotted lines in (a), (b). Scalebars in (a),v(b): $10 \mu \mathrm{m}$. 
Fig. S5, Effects of rotation of the excitation side 2D galvo scan pattern.

\section{Excitation Side 2D Galvo}
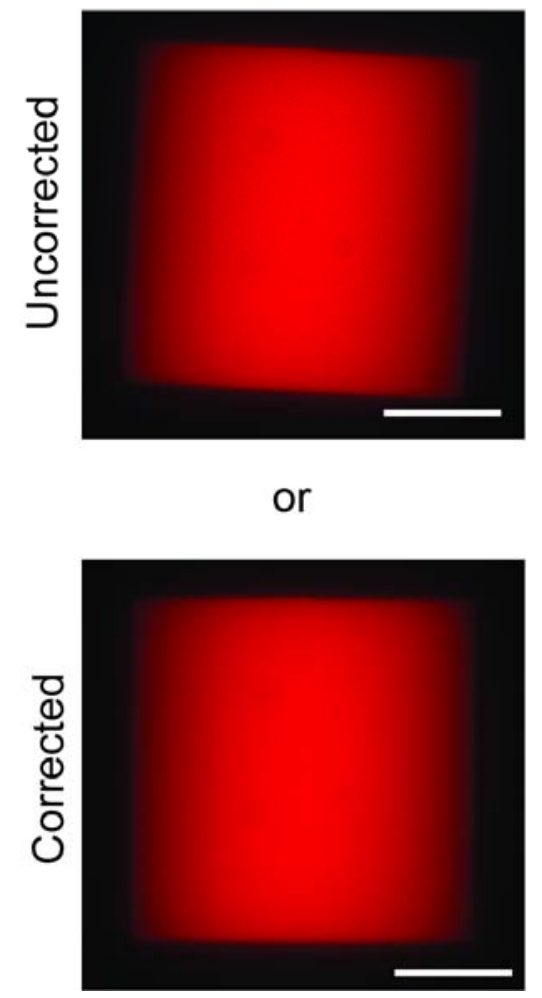
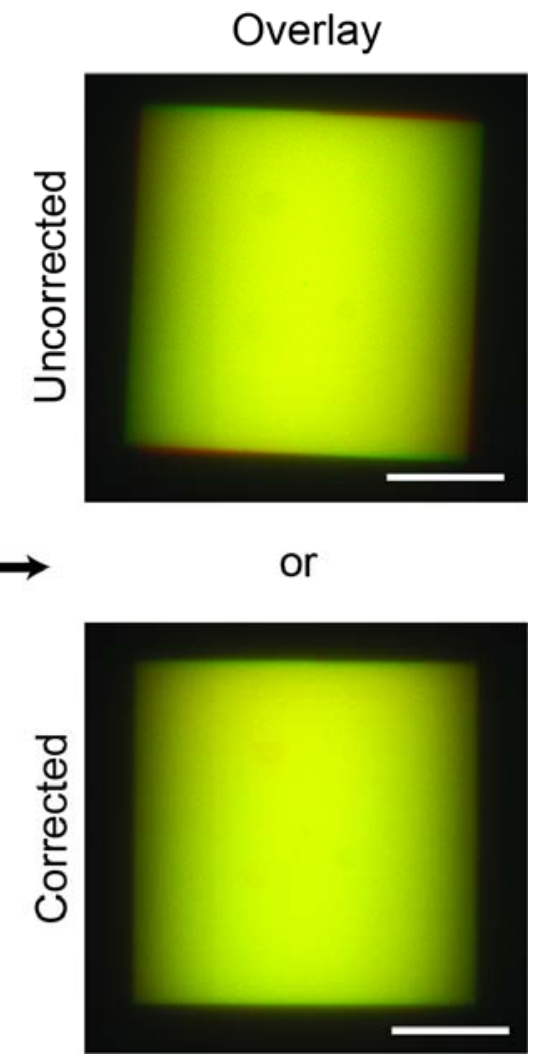

2D scans of a square region of a thick fluorescent lake by either the excitation- or emission-side galvo assemblies show a slight mismatch in the alignment of the scan patterns. This is corrected by rotating the orientation of the excitation side galvo assembly cartesian coordinate scan pattern by $2.81^{\circ}$ according to $x^{\prime}=x \cos \theta-y \sin \theta$ and $y^{\prime}=x \sin \theta+y \cos \theta$. Scale bars: 10 $\mu \mathrm{m}$. 
Fig. S6, Optimization of the phase offset between the excitation- and emission-side fast galvanometric mirrors.

(a)
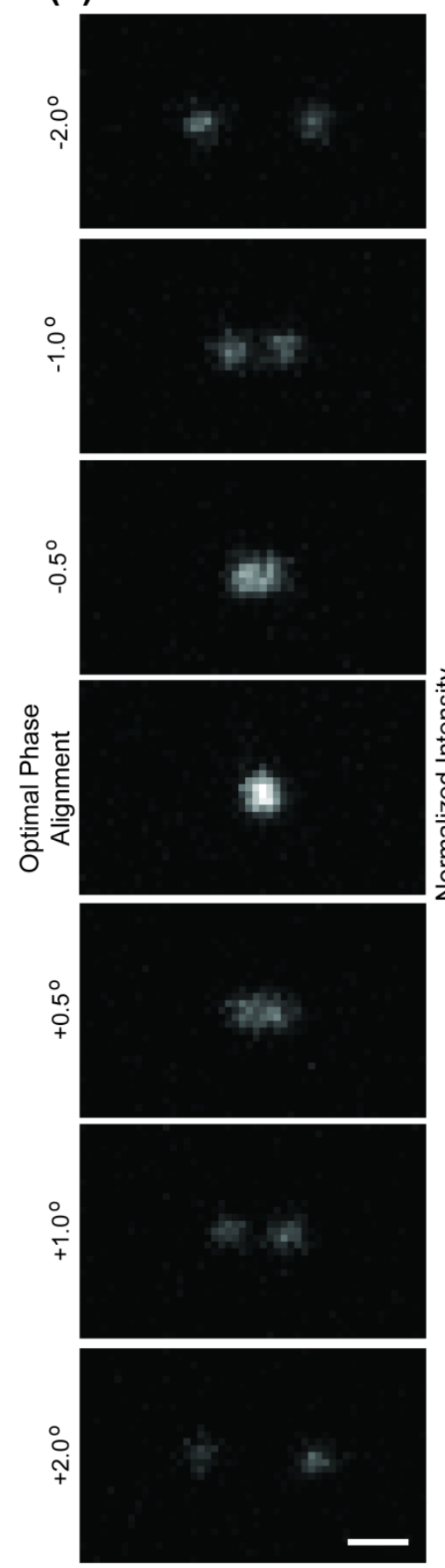

(b)
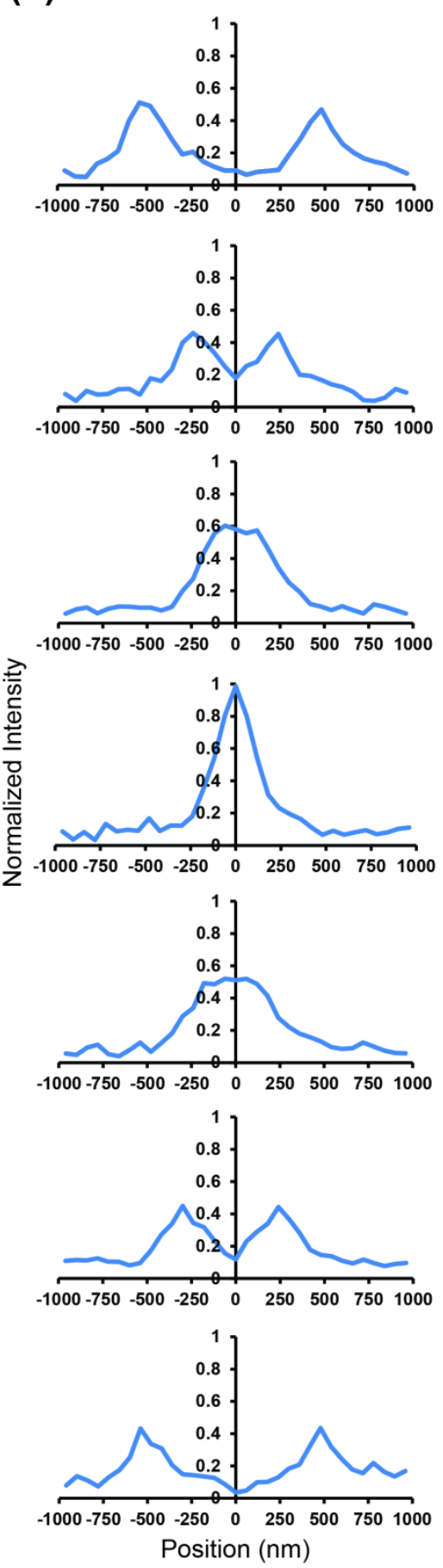

Achieving optimal imaging in 2P ISIM requires setting the correct phase offset between the excitation- and emission-side fast galvo mirrors. Adjusting this phase offset is facilitated by visually monitoring the appearance of a single bead: the bead will appear as a singlet only when the two mirrors move in phase. Incorrect phase offsets will produce a 'splitting', eventually causing the bead to appear as a doublet. (a) Images as a function of phase offset; (b) line-outs through the center of the bead to accompany images. Scalebar: $500 \mathrm{~nm}$. 
Fig. S7, Immunolabeled microtubules viewed in 2P ISIM.
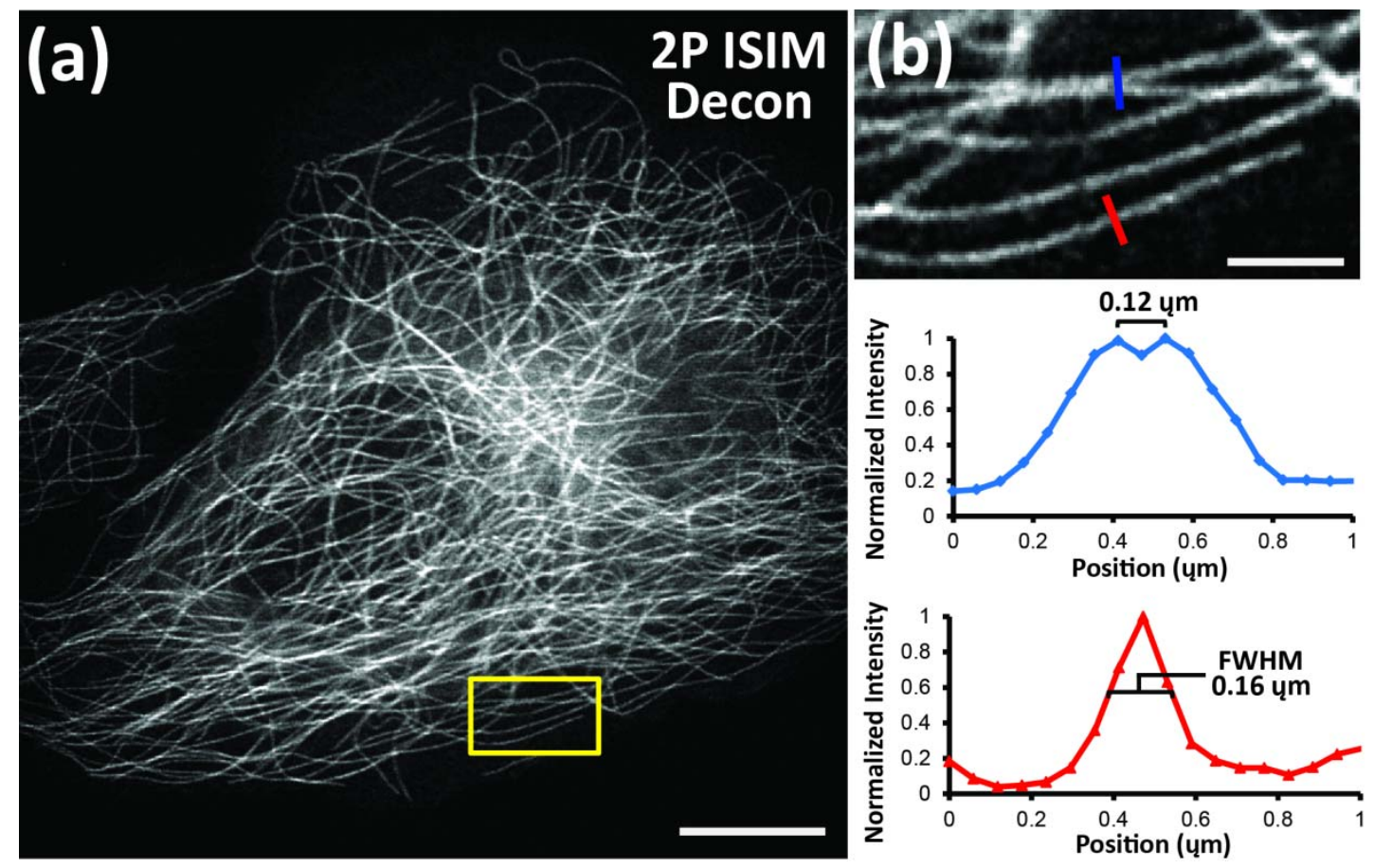

(a) Immunolabeled microtubules in a fixed U2OS human osteosarcoma cell, as viewed in 2P ISIM, after deconvolution. (b) Higher magnification view of the blue rectangular region in (a). Line-outs of microtubules marked in blue and red in are also shown, emphasizing apparent microtubule separation and width. Scalebars: $10 \mu \mathrm{m}$ in (a), $2 \mu \mathrm{m}$ in (b). The cell is the same as in Fig. $\underline{2 .}$ 
Fig. S8, Apparent size of subdiffractive fluorescent beads using different imaging methods.

(a)

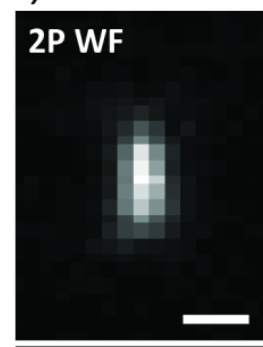

2P WF Decon

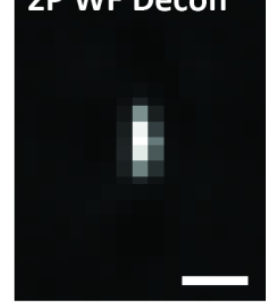

\section{P ISIM}
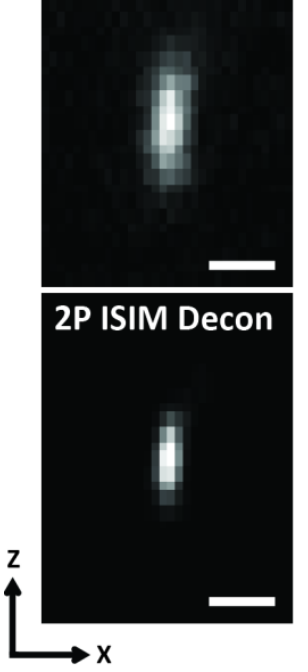

(b)
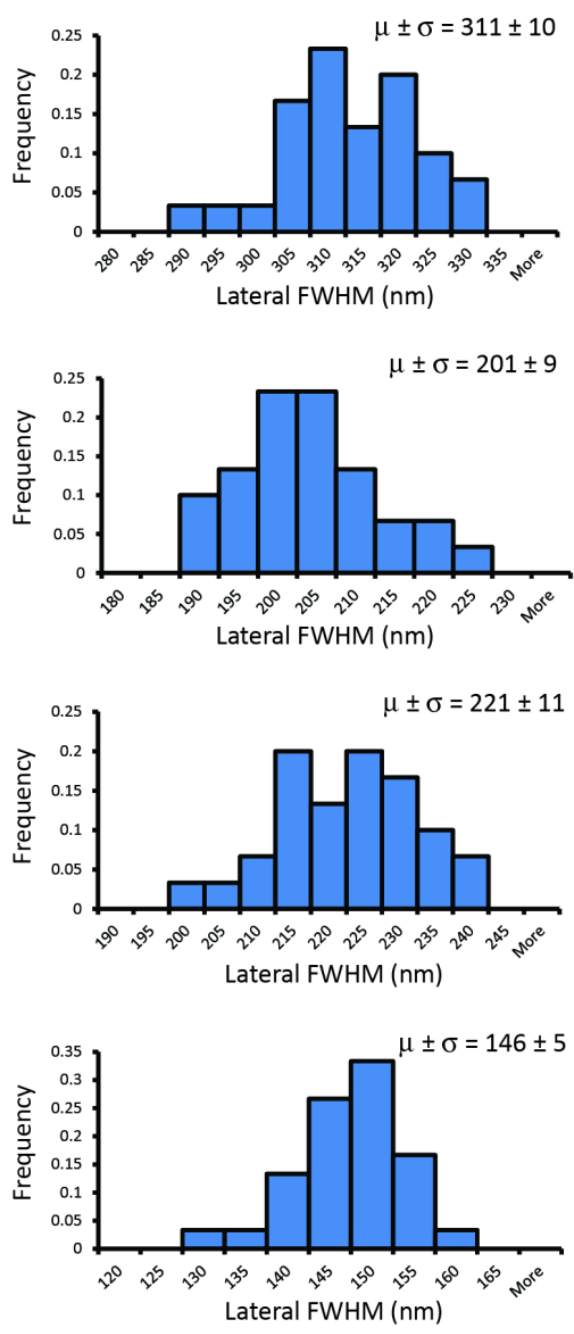

(c)
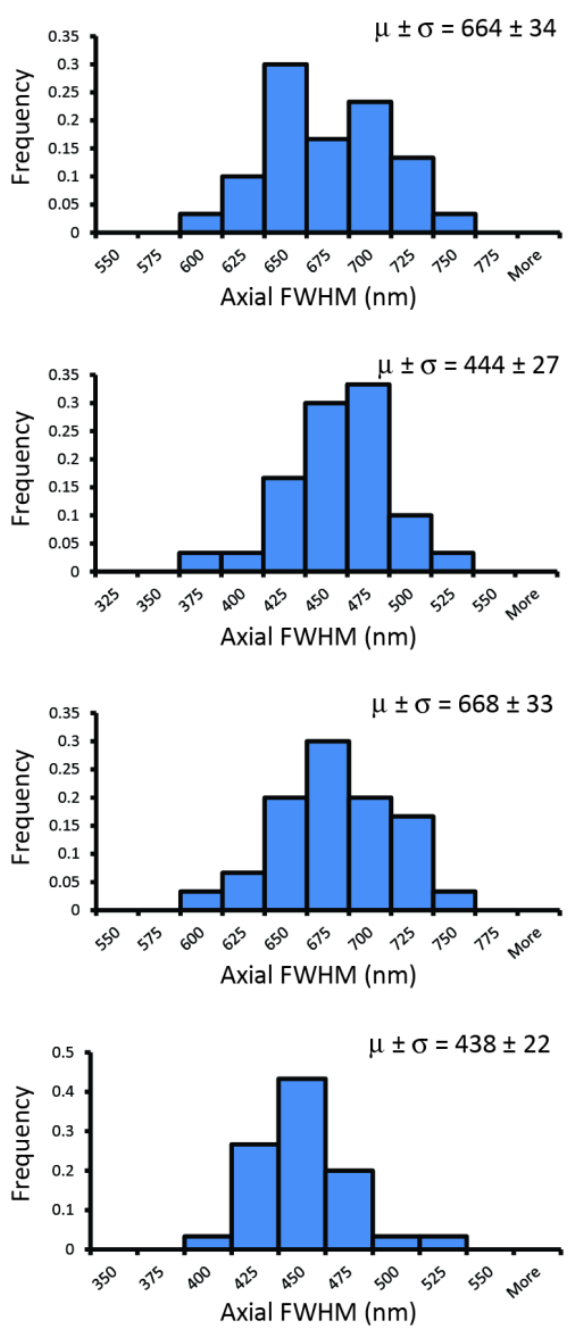

(a) Cross-sectional views through a representative subdiffractive bead for all four imaging conditions, highlighting the difference in resolution between various imaging modes. 2P ISIM improves lateral, but not axial resolution compared 2P excitation, widefield detection imaging (2P WF). Deconvolution improves lateral and axial resolution in all cases. Corresponding histograms of lateral (b) and axial (c) resolution from 30 beads, with means $(\mu)$ and standard deviations $(\sigma)$ indicated. Scalebars: $500 \mathrm{~nm}$. 
Fig. S9, Comparison of degradation in signal and background as a function of depth in scattering samples for different SIM systems.
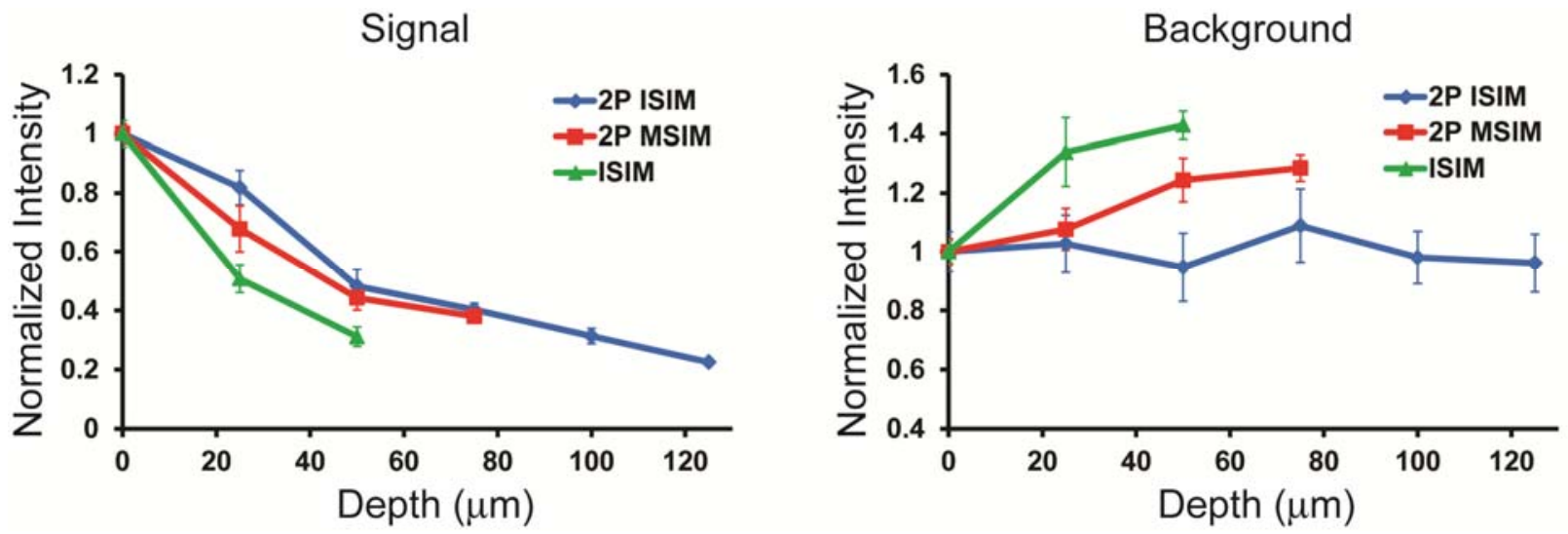

Signal (left) and background (right) were measured for different SIM systems at increasing distances from the coverslip, by imaging fluorescent beads in scattering gels. 2P ISIM offers higher signal levels and lower background at depth than does $1 \mathrm{P}$ ISIM or 2P MSIM. Signal and background levels were normalized to those measured at the coverslip. See also Fig. $\underline{3}$. 
Fig. S10, Axial sectioning of different SIM systems.

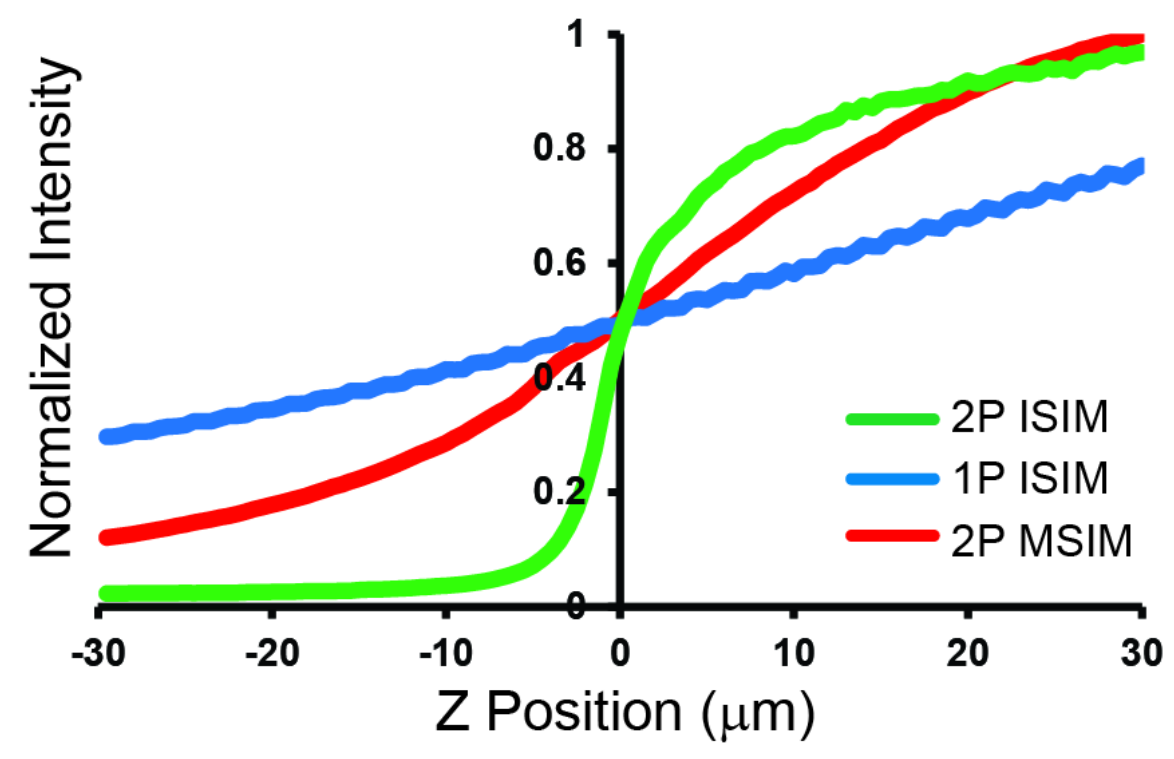

One photon instant SIM (blue), two photon multifocal SIM (red), and two photon instant SIM (green) were used to measure the axial response to a thick fluorescent lake over a range of $60 \mu \mathrm{m}$. The lake was moved axially and the fluorescence integrated at each position. The $0 \mu \mathrm{m} \mathrm{Z}$ position corresponds to the surface of the thick fluorescence lake. Limitations in the piezo stage for the 2P MSIM system prevented us from measuring the response of this system over a range greater than 60 ?m. For 1P ISIM and 2P ISIM data were acquired over a much larger range; only a selected subset of the data is shown here. Data have been normalized: an intensity value of ' 1 ' corresponds to the maximum intensity present in the data, an intensity value of ' 0 ' corresponds to the signal recorded with the lights off and no excitation. $2 \mathrm{P}$ ISIM offers the best sectioning performance. 
Fig. S11, Nematode embryos as viewed in 1P ISIM.

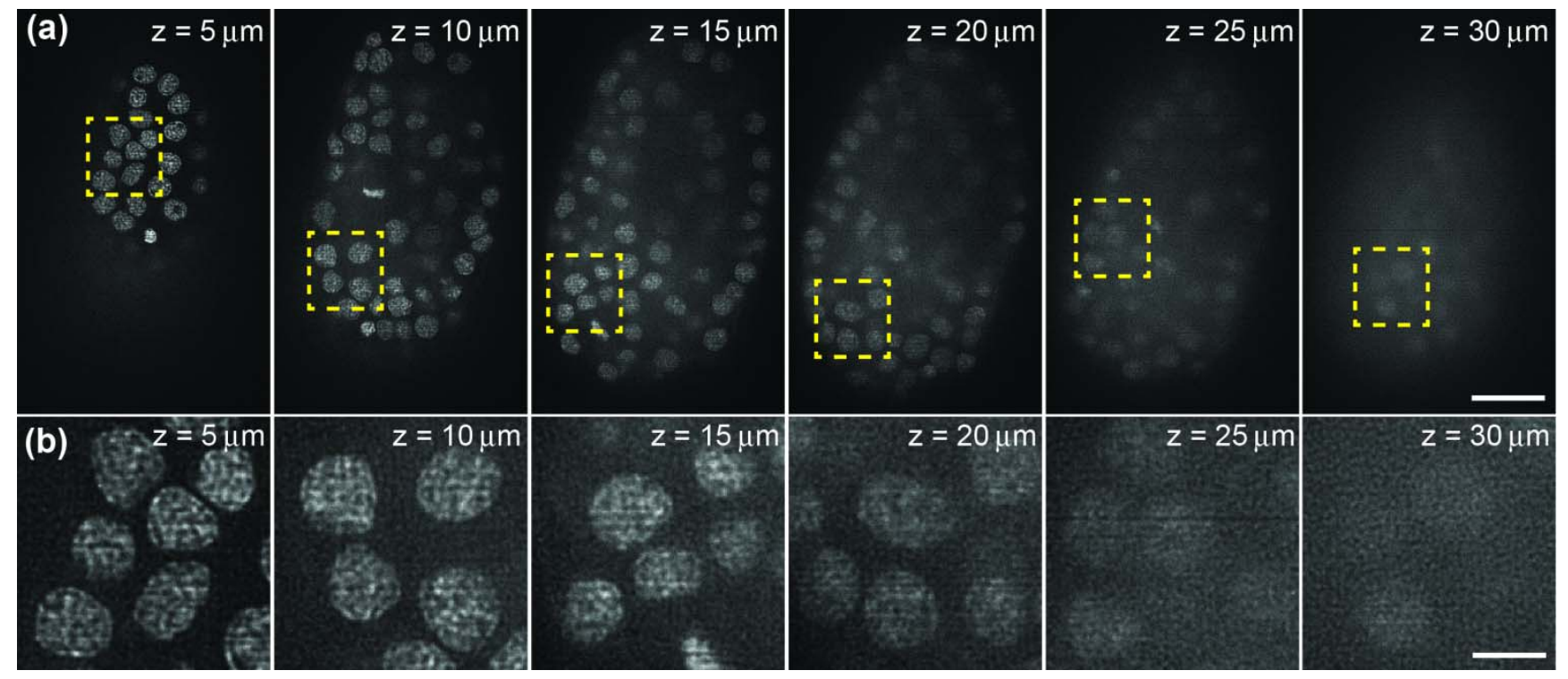

A nematode embryo expressing GFP-H2B in nuclei, at a similar developmental stage as presented in Fig. 4 , but taken using 1P ISIM. (a) XY slices of the worm embryo at indicated axial position from the coverslip. Scalebar: $10 \mu \mathrm{m}$. (b) Higher magnification views of the yellow rectangular regions in (a) Scalebar: $3 \mu \mathrm{m}$. Note the loss in contrast at increasing axial depths. 
Fig. S12, Increasing the exposure in 1P ISIM does not significantly improve imaging quality when examining nematode embryos.

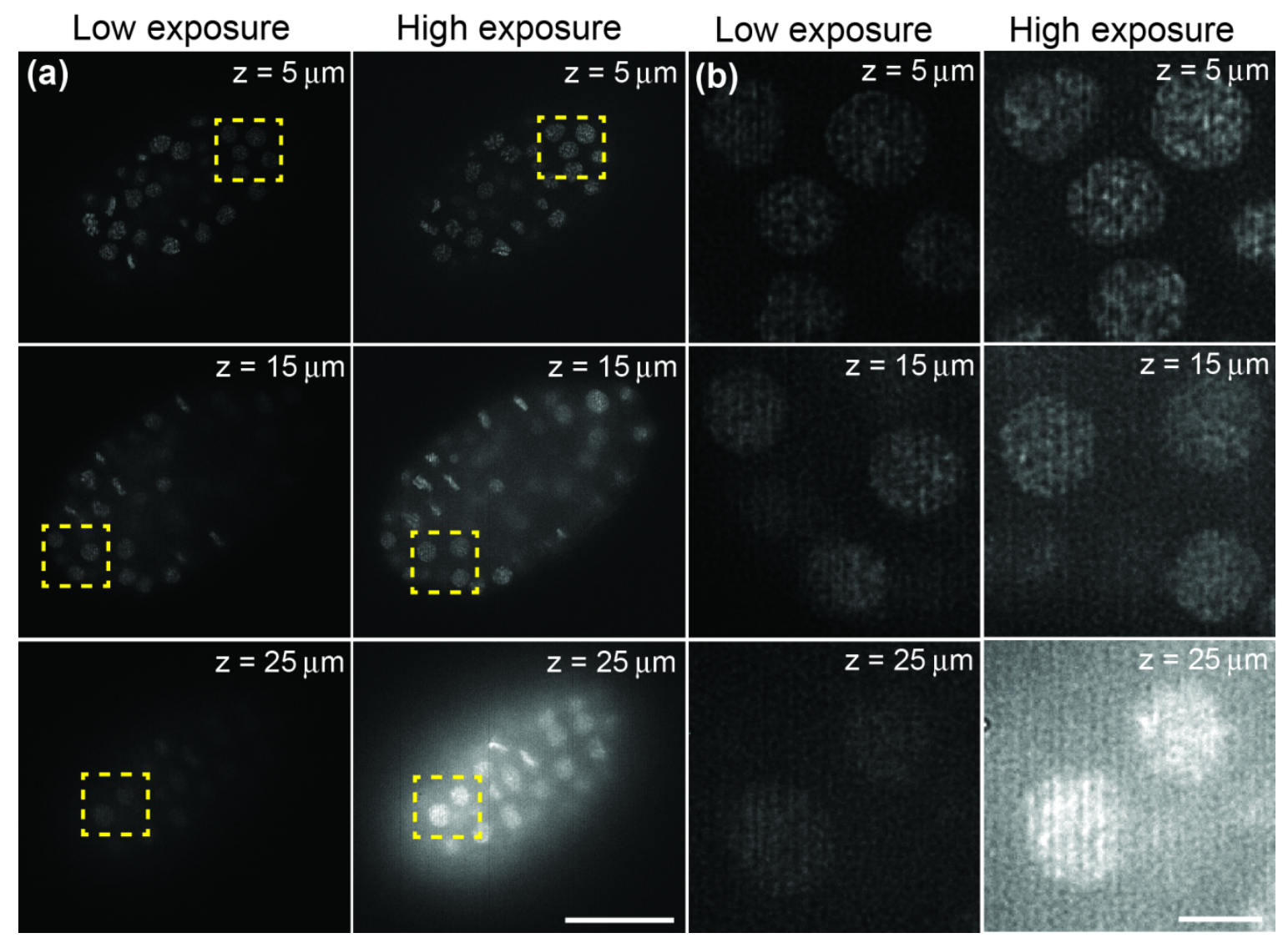

The same embryo was compared at low (first and third columns) and 10x higher exposure (second and fourth columns). Using higher exposures increases both signal and background without affecting resolution loss due to depth-dependent aberrations and scattering. The intensity scale was held constant in all images, and higher magnification views of the yellow rectangular regions in (a) are presented in (b). Scalebars: (a), $20 \mu \mathrm{m}$; (b), $3 \mu \mathrm{m}$. 
Fig. S13, Degradation of axial resolution in zebrafish embryos as a function of depth.

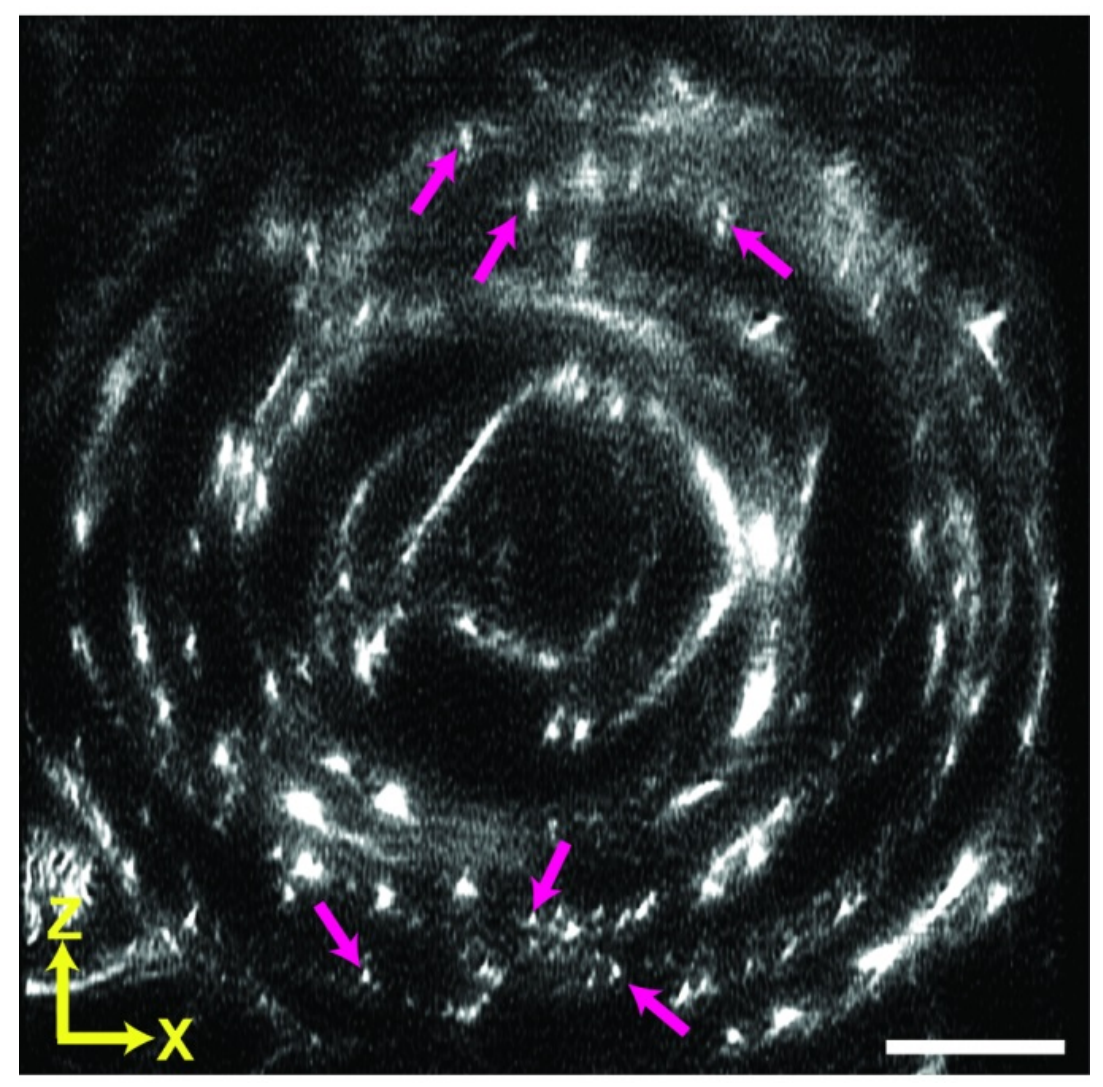

Image is identical to Fig. $6 \mathrm{~g}$, but several filament bundles have been highlighted (magenta arrows) near the bottom and top of the stack. Filament bundles appear small in axial extent near the coverslip, but appear larger as a function of increasing depth, presumably due to depth-dependent aberrations. Scale bar: $20 \mu \mathrm{m}$. 
Table S1, Acquisition parameters for all 2P ISIM images.

\begin{tabular}{|c|c|c|c|c|c|}
\hline Sample & Figure & $\begin{array}{c}\text { Frame } \\
\text { Averaging }\end{array}$ & $\begin{array}{c}\text { 2D Slice Acquisition } \\
\text { Time }\end{array}$ & $\begin{array}{c}\text { Z-step } \\
\text { size }\end{array}$ & Total Axial Range \\
\hline Fixed U20S cells & $1, \mathrm{~S} 8$ & 8 & $4.8 \mathrm{~s}$ & $200 \mathrm{~nm}$ & $2 \mu \mathrm{m}$ \\
\hline $\begin{array}{c}\text { Fluorescent bead } \\
\text { layer }\end{array}$ & $\mathrm{S} 7$ & 4 & $2.4 \mathrm{~s}$ & $\mathrm{NA}$ \\
\hline $\begin{array}{c}\text { Fluorescent bead } \\
\text { layer }\end{array}$ & $\mathrm{S} 9$ & 12 & $4.8 \mathrm{~s}$ & $100 \mathrm{~nm}$ & $3 \mu \mathrm{m}$ \\
\hline $\begin{array}{c}\text { Fluorescent beads in } \\
\text { scattering gel }\end{array}$ & $2, \mathrm{~S} 10$ & 8 & $0.6 \mathrm{~s}$ & $100 \mathrm{~nm}$ & $60 \mu \mathrm{mm}$ \\
\hline $\begin{array}{c}\text { Thick fluorescent } \\
\text { lake }\end{array}$ & $\mathrm{S} 11$ & 1 & $4.8 \mathrm{~s}$ & $250 \mathrm{~nm}$ & $35 \mu \mathrm{m}$ \\
\hline C. elegans embryo & 3 & 8 & $4.8 \mathrm{~s}$ & $500 \mathrm{~nm}$ & $20 \mu \mathrm{m}$ \\
\hline $\begin{array}{c}\text { C. elegans L2 larva } \\
\text { (Both channels) }\end{array}$ & 4 & 8 & $3.0 \mathrm{~s}$ & $250 \mathrm{~nm}$ & $110 \mu \mathrm{m}$ \\
\hline $\begin{array}{c}\text { Zebrafish embryo } \\
\text { (eyeball) }\end{array}$ & $5, \mathrm{~S} 14$ & 5 & $3.0 \mathrm{~s}$ & $250 \mathrm{~nm}$ & $70 \mu \mathrm{m}$ \\
\hline $\begin{array}{c}\text { Zebrafish embryo } \\
\text { (midbrain) }\end{array}$ & 6 & 5 & & & \\
\hline
\end{tabular}




\section{Note S1, Inherent and computational sectioning in SIM.}

Both 3D SIM and confocal microscopy achieve optical sectioning (i.e. the removal of out-of-focus light), albeit by different mechanisms. Previous 3D SIM uses computation to achieve optical sectioning. As noted by Gustafsson [1], the purely computational removal of out-of-focus background does not remove the shot noise associated with this background, whereas the physical rejection of background afforded by the pinhole in confocal microscopy does, in so far that this background never reaches the detector. In thickly fluorescent samples where the in-focus emission would otherwise be swamped by emission originating from outside the focal plane, the optical sectioning mechanism of confocal microscopy is thus strictly superior to the computational optical sectioning provided in 3D SIM.

By using the 2P excitation, out-of-focus emissions are largely eliminated to begin with. 2P ISIM thus achieves a similar result to confocal microscopy, but without the need for a pinhole. In this sense, the optical sectioning in 2P ISIM is also inherent to the microscope and is not the result of computational processing as in previous 3D SIM. As such, it also offers superior background rejection capability relative to purely computational methods

1. M. G. L Gustafsson, L. Shao, P. M. Carlton, C. J. R. Wang, I. N. Golubovskaya, W. Z. Cande, D. A. Agard, J.W. Sedat, “Threedimensional resolution doubling in wide-field fluorescence microscopy by structured illumination, Biophys. J. 94, 4957-4970 (2008). 


\section{Note S2, Explanation of expansion factor choice.}

Here we explain why we chose an expansion factor of $2 \mathrm{x}$ in our system (i.e. scanning the emission-side galvo so that adjacent emission foci are separated by twice the distance they would be if no emission galvo was used). A theoretical expression for the lateral width $W$ (FWHM) of the spot after re-scanning, assuming Gaussian PSFs, is given by1:

$$
W^{2}=\left(\frac{W_{e m}}{M}\right)^{2}+\left(\frac{(M-1) W_{e x}}{M}\right)^{2}
$$

Where $W_{e m}$ and $W_{e x}$ are the widths of the emission and excitation PSFs and $M$ is the scan pattern expansion factor, i.e. the factor by which the distance between adjacent emission foci is increased using the emission-side galvo. In 1P point-based SIM implementations, where the size of the excitation and emission PSFs are approximately equal, this theory predicts that $W$ is minimal when $M=2$ (Figure SN2, red curve).

Fig. SN2, Theoretical dependence of resolution on expansion factor

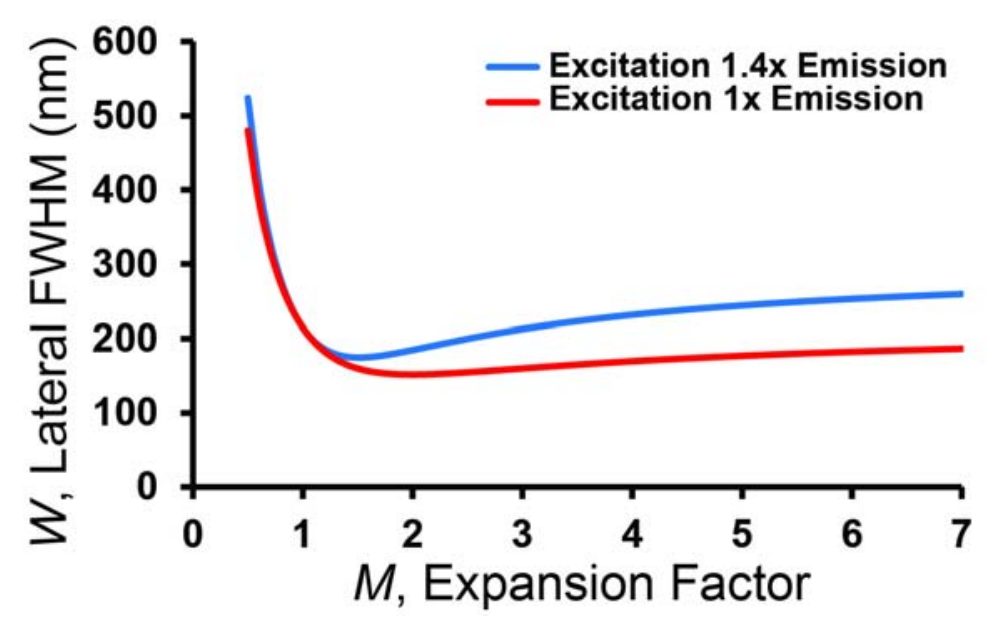

The lateral FWHM $W$ is plotted as a function of expansion factor $M$, assuming the excitation PSF is $1.4 \mathrm{x}$ the size of the emission PSF (blue curve) or the same size as the emission PSF (red curve). Values were calculated assuming an objective NA of 1.2 and an emission wavelength of $515 \mathrm{~nm}$.

However, for 2P illumination where the excitation wavelength is twice the emission wavelength, the expected size of $W_{e x}$ is $\sim 1.4 \mathrm{x}$ larger than $W_{e m}$ (2x larger due to the doubling in wavelength, $1.4 \mathrm{x}$ smaller due the squaring effect of $2 \mathrm{P}$ excitation). The above equation then implies that $W$ is minimal when $M=1.5$ (although we note that setting $M=2$ does not degrade the theoretical resolution too much in this case, blue curve in Fig. SN2).

Interestingly, when imaging $100 \mathrm{~nm}$ subdiffractive fluorescent beads using different expansion factors, we determined that in fact, setting $M=2$ gave better results than setting $M=1.5$. For example, the apparent lateral FWHM (predeconvolution) using an expansion factor of 1.5 was $274 \pm 14 \mathrm{~nm}$ (mean $\pm \mathrm{sd}, N=10$ beads). While improved compared to setting $M=1$ (lateral FWHM: $311+/-10 \mathrm{~nm}$ ), this value is significantly worse than the value that results when setting $M=2$ (lateral FWHM: $221+/-11 \mathrm{~nm}$ ). Additionally, measurements of our 2P excitation (lateral FWHM: $370+/-33, N=10$ beads) and emission (lateral FWHM: $319+/-10 \mathrm{~nm}, N=10$ beads) PSFs revealed that they are roughly equivalent, supporting the choice of $M=2$.

1 G. M. R. De Luca, R. M. P. Breedijk, R. A. J. Brandt, C. H. C. Zeelenberg, E. de Jong Babette, W. Timmermans, L. Nahidi Azir, R. A. Hoebe, S. Stallinga, E. M. M Manders, "Re-scan confocal microscopy: scanning twice for better resolution," Biomed Opt Express 4, 2644-2656 (2013). 